\title{
Revisiting Strong Assumptions in Psychological Constructs Measurement: Further Studying Deviations from Unidimensionality and Bias in Latent Variable Measurement Models Estimates
}

\author{
Gordana Rajlic
}

In the realities of measurement in social and behavioural sciences, in addition to the characteristic(s) of the respondents targeted by the measurement, other influences (other characteristics of the respondents and the items) can be reflected by the responses to the items in a measure. In the current study, different levels of deviations from strict unidimensionality in measures and the accuracy of parameter estimates of widely used unidimensional latent variable measurement models were further investigated. Of interest were unidimensionality violations in measures intended/designed as unidimensional (when the items primarily reflect a dominant latent dimension, as intended in a unidimensional measure, but also reflect, to a smaller degree, some additional influences). In the simulated conditions of interest, varying degrees of systematic error (bias) in the unidimensional model item and person parameters estimates were demonstrated (e.g., factor loadings overestimation and measurement error underestimation). The strength of the relevant relations and the size of bias were examined. If the size of these systematic distortions is uncommunicated, various negative consequences can ensue for substantive research and applied measurement (in relation to the reliability, validity, and fairness of research/measurement outcomes), when the model estimates are used. The utility of the approach employed in the study was discussed.

Keywords: factor analysis, IRT, item/person parameters, systematic measurement error, unidimensionality assumption.

Latent variable measurement models (or common factor models), such as factor analytic (FA) and item response theory (IRT) models (e.g., Birnbaum, 1968; Bock, 1972; Jöreskog, 1969; Lord, 1952; Lord \& Novick, 1968; Rasch, 1960; Samejima, 1969) are widely used in measurement and research in psychology and other social and behavioural sciences. The models estimates of item parameters (e.g., item discrimination/factor loadings) and person parameter (trait/ability) are utilized for many different purposes, in applied research and in practical measurement contexts. In applied research, predominantly used are FA models (such as confirmatory factor analysis models), while IRT models have been traditionally more employed for practical measurement and assessments (e.g., in educational assessment, with increasing use in other domains of psychology, social, and health sciences measurement).

Gordana Rajlic, https://orcid.org/0000-0002-3226-7610. Correspondence to: grajlic@ gmail.com 
Many models have been developed, and they differ in many important aspects; however, of importance for all traditional unidimensional latent variable models are the assumption of unidimensionality - the assumption that a single latent dimension underlies the responses on the given measure, and the assumption of local independence (LI) or conditional independence - the assumption that different items responses are independent conditioning on the underlying latent traits. In unidimensional models, LI actually defines unidimensionality (Birnbaum, 1968; Lord \& Novick, 1968; McDonald, 1981); specifically, a set of item responses is unidimensional when the item responses are locally independent based on a single latent trait. Hence, violations of LI indicate that strict unidimensionality is, to a certain degree, violated too. In its different forms stronger or weaker, the assumption of LI is essential for traditional latent variable measurement models.

In real life measurements, a strong form of LI assumption cannot be met, and certain violations of strict unidimensionality are always present in the responses to the items in a measure (Hambleton \& Swaminathan, 1985; McDonald, 2000; Nandakumar, 1991; Stout, 1987; Traub, 1983). A relaxed form of LI assumption (e.g., essential independence that defines essential unidimensionality; Stout 1987) is also a matter of degrees. Further examining and providing more details about deviations from strict unidimensionality and the accuracy of unidimensional latent-variable models parameter estimates was the general purpose of the current project. The study was conducted in the IRT framework (with a particular IRT model, two-parameter IRT model), with the addressed issues relevant in the item FA context.

Violations of unidimensionality and the consequences of such violations have been investigated in a number of studies (including Ackerman, 1989; Ansley \& Forsyth, 1985; Bonifay, Reise, Scheines, \& Meijer, 2015; Crişan, Tendeiro, Meijer, 2017; Drasgow \& Parsons, 1983; Kahraman, 2013; Kirisci, Hsu \& Yu, 2001; Reckase, 1979; Zhang, 2008). In a related line of research, violations of LI were studied (including Chen \& Thissen, 1997; DeMars, 2012; Sireci, Thissen, \& Wainer, 1991; Wainer \& Thissen, 1996; Yen, 1993). The findings of the majority of the studies supported the conclusion about robustness of the unidimensional models to unidimensionality violations. That is, a general conclusion was supported that small violations do not lead to serious consequences in terms of the accuracy of model parameter estimates. More specific information and recommendations about the conditions of robustness were stated in different ways in the studies, depending on how the violations of unidimensionality were conceptualized and manipulated. The violations were conceptualized and manipulated in many different ways, and this heterogeneity is related to challenges in summarizing the findings, and the absence of clear, widely-accepted recommendations for model users (Kahraman, 2013; Kirisci et al., 2001; Ip, 2010).

Most often studied in research about robustness of unidimensional models were violations of unidimensionality consistent with "substantive" multidimensionality in a measure, when more than one interpretable, meaningful factor can be identified underlying item responses (Ackerman, 1989; Ansley \& Forsyth, 1985; Kirisci et al., 2001). Another type of multidimensionality in items and measures and its consequences have been emphasized in the recent research (Ip, 2010; Kahraman, 2013; Reise, et al., 2013; Zhang, 2008). Specifically, a need for further investigation of the "weaker" forms of unidimensionality violations, when smaller, secondary dimensions underlie item responses, in addition to the dominant one, was 
highlighted. This line of research is closely related to the line of research about violations of LI/essential independence and their effects, as by definition, such violations manifest as secondary dimensions underlying item responses (Lazarsfeld, 1950; Stout, 1987).

Secondary dimensions in measures can arise for many different reasons. Item pairs, item groups, or all the items get tied together by a certain common theme, reflecting various other influences in addition to the construct they were intended to measure. Some items, for example, can bundle together due to referring to the same material (e.g., a paragraph in the test), due to wording style (e.g., negative worded items), or various other method effects. Or, in another scenario, some of the items in the measure may tap into other related constructs, in addition to the target construct, but to a smaller degree. Many reasons for the item clusters, and the ways to deal with the clusters, were addressed in the research on LI violations, in educational settings as well as in psychological measurement, in Chen and Thissen (1997); DeMars (2012); Morin, Arens, Tran, and Caci (2016); Reise et al. (2007); Steinberg and Thiessen (1996); and Yen (1993).

Some of the weaker forms of unidimensionality violations have been studied less. For instance, Traub (1983) provided examples of the influences that differentiated between different groups of respondents, and when their responses were combined, inter-item dependencies remained after the target construct was accounted for. Traub discussed, in an educational measurement context, the effects of propensity to guessing, responding speed, or receiving additional training, on unidimensionality of items and measures. It can be inferred that, in the same way, systematic differences in many other respondents' characteristics can influence the undimensionality of a measure. They could manifest as residual item dependencies and secondary dimensions in measures (as opposed to substantive, interpretable factors identified in FA).

In real life measurement, when deviations from strict unidimensionsionality are identified in a unidimensional measure, it may be difficult to pinpoint what exactly is behind such violations and what else our items could be tapping into. In relation to this, the possible nature of violations of unidimensionality in measures has been debated often, in various measures and measurement contexts (e.g., Alessandri, Vecchione, Eisenberg, \& Łaguna, 2015; Marsh, Scalas, \& Nagengast, 2010; Rajlic, Kwon, Roded, \& Hubley, 2019). An approach that could be useful for providing further information about the effects of violations of strict unidimensionality due to a range of unintendedly captured influences was explored in the current study.

\section{Current Study}

The current study was conducted to further explore different levels of violations of strict unidimensionality in measures and the accuracy of the unidimensional latent variable models parameter estimates in such conditions. A particular type of violations was of interest, in measures intended and designed as unidimensional. Specifically, the conditions of violations were simulated when all of the items in a measure primarily capture a single dominant latent dimension (as intended in a unidimensional measure), but additionally, to a smaller degree, they tap into some additional influences. Some of the characteristics of the items (e.g., various "method effects") or characteristics of the respondents (including background characteristics and various personality traits/abilities irrelevant for the purpose of measurement) could influence items responding and lead to unidimensionality violations not manifesting as interpretable, 
substantive multidimensionality in measures. A particular model and study design, as described in the Method section were proposed as useful for studying the effects of different levels of violations of strict unidimensionality in the measures intended as unidimensional. In the different composites of a dominant latent dimension and some additional influences simulated, the unidimensional model item and person parameters estimates were examined.

For technical convenience, the study was conducted in the IRT framework, and the estimates of a 2PL IRT model were assessed. Because of the mathematical relation between the two-parameter IRT models and FA of item responses (Takane \& de Leeuw, 1987; Wirth \& Edwards, 2007), the problems addressed, research methodology, and the findings are readily converted into item FA context and FA parametrization, and they are relevant to researchers working with the FA models. The conditions relevant for substantive research in social and behavioral sciences (rather than large-scale assessments and educational measurement) were given greater consideration in the study design.

\section{Model and Study Design}

\section{Method}

To depict the conditions of unidimensionality violations of interest in the current study, a multidimensional model was used with two latent dimensions: a) a dominant dimension, with all items primarily tapping into this dimension", and b) a secondary "nuisance" dimension, representing source(s) of item dependencies remaining after the dominant trait was accounted for. In this model, the items loaded on more than one simulated dimension, that is, complexstructure items were of interest in the study. The complex-structure items were described as items that do not share only one common attribute (McDonald, 1999) - in addition to reflecting the target construct, the item responses reflect something else as well. The resulting multidimensionality was characterized as within-item multidimensionality, as opposed to between-item multidimensionality ${ }^{3}$.

To depict violations in measures intended and designed as unidimensional, the design was utilized in which the strength of the two simulated dimensions was systematically varied in a particular way. Following the FA tradition, the strength of the latent dimensions was defined in the study by the strength of the relations between the items and the dimensions (i.e., by magnitude of item discrimination parameter in IRT parametrization or factor loadings in FA parametrization). The magnitude of the item discrimination on the two dimensions was varied and several combinations of the strength of the dimensions were created, with the secondary

\footnotetext{
${ }^{2}$ The dominant trait tapped by all items can be, in unidimensional latent variable framework, presumed as the target trait - the construct that the measure was intended/designed to measure.

${ }^{3}$ In studying violations of unidimensionality and its consequences, items that are restricted in a measurement model to discriminate on only one of the dimensions and corresponding between-item multidimensionality, have been utilized predominantly, with the greater attention to the complex items responses and within-item multidimensionality dedicated to in the more recent research (e.g., Kahraman, 2013; Reise, et al., 2013; Zhang, 2008).
} 
dimension of less strength in all of the conditions compared to the dominant one. For example, condition 1 (very strong - negligible) was a combination of a very strong dominant dimension (very high item discrimination on that dimension) and a negligible secondary dimension (very low item discrimination on that dimension), as described in the section about simulated conditions. In the conditions of interest, the unidimensional model estimates were examined in relation to the target construct (i.e., the dominant dimension). The contribution of the strength of the dominant dimension and the strength of the secondary dimension to the distortions in the unidimensional model item and person parameter estimates was explored.

The model and the design utilized in the study combines the concepts of locally-dependent unidimensional models and of essential unidimensionality (Humphreys, 1964; Ip, 2010, Nandakumar, 1991; Stout, 1987). The secondary dimension in the model was conceptualized in a very general way, as residuals, the item dependencies not accounted for by the dominant dimension (without focusing on some specific sources and reasons for such dependencies), and therefore uncorrelated with the dominant dimension ${ }^{4}$. A single secondary dimension was proposed to represent additional influences affecting item responses: Increasing strength of the secondary dimension (i.e., an increase in the conditional inter-item correlations) indicates that the items are increasingly capturing "something else" - a single, or a group of correlated additional influences. The model with a single secondary dimension is simpler and more convenient than the other multidimensional models (e.g., multidimensional models with several secondary dimensions, and hierarchical/bifactor models with several group factors), and it was proposed as sufficient for the violations of unidimensionality in measures intended as unidimensional, when further distinguishing among possible different sources of constructirrelevant variance is not of interest. A range of scenarios for weaker forms of unidimensionality violations in measures could be represented in this way. Ip (2010) demonstrated that locallydependent unidimensional models and multidimensional models are empirically indistinguishable; therefore, multidimensional IRT model (MIRT; Reckase, 2009) was used for the data simulation in the current study.

\footnotetext{
${ }^{4}$ The model with uncorrelated dimensions, as opposed to predominantly utilized "correlated-traits model", was posited. The model with correlated dimensions (correlated to a certain degree, depending on what specific constructs are in question) is more appropriate in the situations pertaining to some particular, interpreted, sources of unidimensionality violations.
} 


\section{Simulated Conditions}

Four levels of strength of the dominant dimension (very strong, strong, moderate, and weak) were crossed with four levels of strength of the secondary dimension (negligible, weak, moderate, and strong). These levels of strength of the dimensions were characterized by different magnitudes of item discriminations, following the guideline about the magnitude of item discrimination provided by Baker (2001): very high item discrimination $(a>1.70)$, high item discrimination ( $a$ range 1.35 - 1.69), moderate item discrimination ( $a$ range 0.65 - 1.34), low item discrimination ( $a$ range 0.35 - 0.64), and very low item discrimination ( $a$ range 0.01 $0.34)^{5}$. Accordingly, a very strong dimension was characterized by very high item discrimination, a strong dimension by high item discrimination, a moderate dimension by moderate item discrimination, a weak dimension with low item discrimination, and a negligible dimension with very low item discrimination.

Given that in the study design the secondary dimension was conceptualized as always of less strength compared to the dominant one (i.e., studying weaker forms of violations in measures intended/designed as unidimensional was of interest), not all pairings of the strength of the dominant and secondary dimensions were plausible. Hence, a "partially-crossed" research design was used, and ten conditions of interest were designed (Figure 1). Condition 1 (a very strong dominant dimension with a negligible secondary dimension) was characterized by very high item discriminations on the first dimension and very low item discrimination on the second dimension (a full description of all the conditions is included in Supplemental Materials).

A set of item responses was then simulated in each condition (using MIRT model and the items and person location parameters described in the following sections), with 100 replications. This number of replications was deemed sufficient for the given study design (i.e., how item/person parameters were generated). About the item format, dichotomous items were simulated. Even though more basic, such items have been used in psychosocial measures in various domains. More basic conditions, including the type of item format (and corresponding model assessed 2PL IRT for binary responses), were addressed in the current study as a good starting point in systematically investigating the given problem. In terms of the number of items, 15 items were simulated - the shorter length was chosen in the study consistent with the general trend towards shorter measures in psychology and social sciences research, when measures are intended as unidimensional. It should be highlighted that the ten conditions are primarily "experimental" conditions (i.e., result of systematic varying of the research variables' levels), designed to investigate the problems of interest (possible distortions in the estimates at different levels of unidimensionality violations). Systematic varying of the variables of interest cannot be achieved

\footnotetext{
${ }^{5}$ The values of item discrimination indexes are in the logistic metric. In FA parametrization (following Wirth \& Edwards, 2007), very high item discrimination, in Baker's classification, in 2PL unidimensional IRT model, corresponds to factor loadings (FL) from categorical confirmatory FA of approximately .71 and greater; high item discrimination corresponds to FL range .62 -.70; moderate item discrimination corresponds to FL range .36 -.61; low item discrimination corresponds to FL range .20 -.35; and very low item discrimination corresponds to FL range of .01 -.19.
} 
with the real measures. In addition to the ten designed conditions of strict unidimensionality violations, four "strictly unidimensional" conditions were simulated: In these conditions, only one dimension was simulated that corresponded to the dominant dimension in the designed research condition (i.e., very strong, strong, moderate, and weak dimension).

DOMINANT DIMENSION STRENGTH

\begin{tabular}{|c|c|c|c|c|c|}
\hline \multirow{5}{*}{ 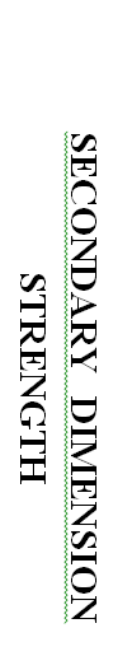 } & \multirow[b]{2}{*}{ NEGLIGIBLE } & $\begin{array}{c}\text { VERY } \\
\text { STRONG }\end{array}$ & STRONG & MODERATE & WEAK \\
\hline & & $\begin{array}{l}\text { 1. Very Strong/ } \\
\text { Negligible }\end{array}$ & $\begin{array}{l}\text { 5. Strong/ } \\
\text { Negligible }\end{array}$ & $\begin{array}{l}\text { 8. Moderate/ } \\
\text { Negligible }\end{array}$ & $\begin{array}{l}\text { 10. Weak/ } \\
\text { Negligible }\end{array}$ \\
\hline & WEAK & $\begin{array}{l}\text { 2. Very Strong/ } \\
\text { Weak }\end{array}$ & $\begin{array}{l}\text { 6. Strong / } \\
\text { Weak }\end{array}$ & $\begin{array}{l}\text { 9. Moderate/ } \\
\text { Weak }\end{array}$ & \\
\hline & MODERATE & $\begin{array}{l}\text { 3. Very strong/ } \\
\text { Moderate }\end{array}$ & $\begin{array}{l}\text { 7. Strong/ } \\
\text { Moderate }\end{array}$ & & \\
\hline & STRONG & $\begin{array}{l}\text { 4. Very strong/ } \\
\text { Strong }\end{array}$ & & & \\
\hline
\end{tabular}

Figure 1. Ten simulated conditions of violations of strict unidimensionality. The conditions were created by combining four levels of strength of the dominant dimension and four levels of strength of the secondary dimension, with the dominant dimension always stronger than the secondary dimension. 100 sets of item responses were simulated for each condition.

\section{Item and Person Parameters Generation}

For the ten conditions of violations of strict unidimensionality, item discrimination parameters ( $a$ parameters), were prescribed by the study design (i.e., based on Baker's, 2001 classification of item discrimination strength), as outlined in the description of the ten created conditions. In each condition, item discrimination parameters were simulated from a normal distribution in such a way that parameter values for the majority of the items belonged to range of values provided by Baker's classification. The mean and standard deviation of the parameter distribution were chosen accordingly. A guide for simulating item discrimination parameters for dominant and secondary dimensions, based on Baker's classification, was provided in Table 1. More detailed descriptions of the item parameter simulation procedure and the actual item parameters simulated for each of the designed conditions and are enclosed in Supplemental Materials.

The same sets of item discrimination parameters were used across multiple designed conditions, for example, the same set of parameters for a very strong dimension was used for the dominant 
dimension in conditions 1, 2, 3 and 4 (see Table 1). This was done in order to keep the focus on the variability resulting from the experimental manipulation (i.e., manipulation of the item discrimination parameters on the two dimension across the conditions) while minimizing the variability resulting from random selection of the parameters within the conditions. Similarly, the same two sets of item location parameters ( $b$ parameters), were used in all the conditions, in order to focus on the variability due to experimental manipulation and minimize variability from any other sources. The two sets of item location parameters were generated from the two successive independent draws from the standard normal distribution, to match the distribution of person location parameters ( $b$ parameters for all items are included in Supplemental Materials).

Table 1: A Guide for Simulating Item Discrimination Parameters for the Ten Designed Conditions

\begin{tabular}{llcc}
\hline \multicolumn{1}{c}{ Designed Conditions } & $\boldsymbol{a}_{1}$ & $\boldsymbol{a}_{2}$ \\
& $M(S D)$ & $M(S D)$ \\
\hline 1. Very strong - Negligible & $1.90(0.20)$ & $0.18(0.17)$ \\
\hline 2. Very strong - Weak & $1.90(0.20)$ & $0.50(0.15)$ \\
3. Very strong - Moderate & $1.90(0.20)$ & $1.00(0.35)$ \\
\hline 4. Very strong - Strong & $1.90(0.20)$ & $1.52(0.17)$ \\
\hline & & \\
\hline 5. Strong - Negligible & $1.52(0.17)$ & $0.18(0.17)$ \\
\hline 6. Strong - Weak & $1.52(0.17)$ & $0.50(0.15)$ \\
\hline 7. Strong - Moderate & $1.52(0.17)$ & $1.00(0.35)$ \\
\hline & & \\
\hline 8. Moderate - Negligible & $1.00(0.35)$ & $0.18(0.17)$ \\
\hline 9. Moderate - Weak & $1.00(0.35)$ & $0.50(0.15)$ \\
\hline & & \\
\hline 10. Weak - Negligible & $0.50(0.15)$ & $0.18(0.17)$ \\
\hline
\end{tabular}

Note: $a_{1}$ - item discrimination on the dominant dimension; $a_{2}-$ item discrimination on the secondary dimension. The $M$ and $S D$ for ' $a$ ' values are chosen based on Baker's (2001) classification of item discrimination strength. The actual parameters generated for 15 items for the ten conditions are enclosed in Supplemental Materials.

Person location parameters for the two dimensions, in the ten designed conditions, were generated from the standard normal distribution for a large sample of respondents $(N=5000)$. Two simulated dimensions were generated as uncorrelated, in accordance with the conceptualization of the model (i.e., the secondary dimension representing item dependencies remaining after the dominant latent trait is accounted for). Person location parameter was treated as random, that is, new parameters were generated across the replications and conditions. The item parameters and person location parameters used in the current study were simulated in $\mathrm{R}$ application (R Development Core Team, 2019). 


\section{Multidimensional Model Used for Data Generation}

To simulate multidimensional data, a two-parameter compensatory MIRT model (Reckase, 2009) was used:

$$
P\left(y_{i, j}=1 \mid \theta_{i}, a_{j}, d_{j}\right)=\frac{1}{1+\exp \left[-\left(a_{j 1} \theta_{i 1}+a_{j 2} \theta_{i 2}+d_{j}\right)\right]}
$$

where the left side of the equation describes the conditional probability that examinee $i$ 's response $y$ to a dichotomous item $j$ is correct, $\theta_{i}$ is latent dimensions vector (with two dimensions $\theta_{i 1}$ and $\theta_{i 2}$ ), $\alpha_{j}$ is item discrimination vector (with $\alpha_{j 1}$ and $\alpha_{j 2}$ corresponding to the two latent dimensions) and $d_{j}$ is the item intercept. Compensatory multidimensional model was chosen as more appropriate for the conceptualization of the two dimensions in the study compared to noncompensatory model. Item parameters and theta parameters, for the two dimensions, and in the ten designed conditions, were generated as described in the previous section. Item responses were simulated in $\mathrm{R}$ application ( $\mathrm{R}$ Development Core Team, 2018), using 'mirt' $\mathrm{R}$ package (Chalmers, 2012).

\section{Unidimensional IRT Model Estimation}

After the sets of item responses were simulated, based on the conditions specified by the study design, unidimensional model estimates were obtained by fitting the unidimensional 2PL IRT model, using R package 'ltm' (Rizopoulos, 2006). The 'ltm' package was chosen as it demonstrated good performance in IRT model parameters recovery studies and it provides estimation of item discrimination/location and person location in $\mathrm{R}$ environment. Marginal maximum likelihood estimation (MMLE) was utilized for estimation of the item parameters and for person location estimation, the Bayesian expected a posteriori (EAP) method was used. These methods are most commonly used for item parameters and person location estimation (de Ayala, 2009).

\section{Outcome Measures}

In the current study, the estimated parameters were item discrimination, item location, and person location values obtained by applying a unidimensional 2PL IRT model to the simulated item responses. The "true" parameters were the item location, item discrimination, and person location values used in the item responses simulation; specifically, the values corresponding to the dominant latent dimension (and the latent dimension in strictly unidimensional conditions). Bias in estimation of item parameters (i.e., item discrimination and item location) was calculated as a difference between the estimated item parameters and the true parameters, averaged across the items, and bias in estimation of person location was calculated as a difference between the estimated person location parameter and the true parameter, averaged across the respondents

$$
\text { bias } \omega=\frac{\sum_{t=1}^{T}(\widehat{\omega}-\omega)}{T}
$$

where $\omega$ is the parameter under consideration (item discrimination, item location, or person location) and $T$ denotes the number of items or number of respondents (depending on if bias in 
item parameters or in person parameter was in question). Overall error (root mean square error, RMSE) in estimation of item parameters was calculated as

$$
R M S E \omega=\sqrt{\frac{\sum_{t=1}^{T}(\widehat{\omega}-\omega)^{2}}{T}}
$$

where $\omega$ is the parameter under consideration (item discrimination, item location, or person location) and $T$ denotes the number of items or number of respondents (depending on if bias in item parameters or in person parameter was in question). Furthermore, bias and RMSE were first assessed in several "strictly unidimensional" conditions, before manipulations according to the study design were performed. In those conditions, only one dimension was simulated that corresponded to the dominant dimension in the designed research condition (i.e., very strong, strong, moderate, and weak dimension), by utilizing the same procedure that was used for simulation of the ten experimental conditions (as described in data generation section). The distortions recorded in the strictly unidimensional conditions are beneficial as a simulation quality check, and as a reference point for interpreting the size of distortions in the unidimensional model estimates obtained in the ten designed conditions.

Bias and RMSE were described and summarized in the designed conditions. The relations between the strength of the two dimensions (the strength of the dominant dimension and the strength of the secondary dimension), and bias/RMSE in the estimation of item discrimination, item location, and person location parameters were summarized. The correlations appropriate for the categorical/ordinal nature of the variables of interest were obtained (i.e., Spearman's correlation coefficients). Semi-partial correlation coefficients were reported, that is, correlations between the strength of one dimension, from which the strength of the other dimension was partialed, and bias ${ }^{6}$.

\footnotetext{
${ }^{6}$ In the case of the dominant dimension, for example, this correlation (i.e., squared correlation coefficient) represents the percentage of total variation in bias associated with the dominant dimension, with the strength of the secondary dimension partialed from the strength of the dominant dimension (but not partialed from the dependent variable, i.e., bias). This way, in the case of both dominant and secondary dimensions, correlations represent the percentage of total variance that each dimension is associated with, making comparison between them feasible.
} 


\section{Item Parameters}

\section{Results}

In relation to item discrimination parameter, bias and RMSE in the unidimensional item discrimination parameter estimates in strictly unidimensional conditions were provided in Table 2. In the ten study conditions (in which the secondary dimension of different strength was introduced), various degrees of bias were documented in the unidimensional estimates of the dominant dimension item discrimination parameter (presented in Table 3). The smallest bias (i.e., closest to strictly unidimensional conditions) was recorded in condition 1 (very strong dominant dimension - negligible secondary dimension) and the largest bias was recorded in condition 4 (very strong dominant dimension - strong secondary dimension). In terms of direction of bias, in all conditions bias was positive, indicating overestimation of the dominant dimension item discrimination parameter in the unidimensional estimates. A graphical presentation of the distribution of bias across replications, in the ten simulated conditions, is included in Supplemental Materials.

A strong relation between the strength of the secondary dimension and bias was demonstrated after controlling for the strength of the dominant dimension, with the magnitude of the correlation of 0.90 ( $N=1000$, i.e., 100 replications in 10 conditions). A weak relation between the strength of the dominant dimension and bias was recorded, with the magnitude of correlation of -0.19 (negative relation - the bias increased with decrease in strength of the dominant dimension), after controlling for the strength of the secondary dimension.

Table 2: Bias and RMSE in the Item Discrimination and Item Location Parameter Estimates of in Strictly Unidimensional Conditions

\begin{tabular}{|c|c|c|c|c|}
\hline \multirow{2}{*}{$\begin{array}{c}\text { Latent Trait } \\
\text { Strength }\end{array}$} & \multicolumn{2}{|c|}{ Item Discrimination } & \multicolumn{2}{|c|}{ Item Location } \\
\hline & $\begin{array}{c}\text { Bias } \\
M(S D)\end{array}$ & $\begin{array}{l}\boldsymbol{R M S E} \\
M(S D)\end{array}$ & $\begin{array}{c}\text { Bias } \\
M(S D)\end{array}$ & $\begin{array}{l}\boldsymbol{R M S E} \\
M(S D)\end{array}$ \\
\hline Very strong & $0.003(0.02)$ & $0.078(0.02)$ & $<0.001(0.02)$ & $0.032(0.01)$ \\
\hline Strong & $0.003(0.02)$ & $0.064(0.01)$ & $<0.001(0.02)$ & $0.041(0.01)$ \\
\hline Moderate & $0.003(0.02)$ & $0.053(0.01)$ & $-0.001(0.02)$ & $0.056(0.01)$ \\
\hline Weak & $0.002(0.01)$ & $0.049(0.01)$ & $0.002(0.03)$ & $0.112(0.03)$ \\
\hline
\end{tabular}

Note: In strictly unidimensional conditions, only one latent trait was simulated that corresponded to the strength of the dominant traits simulated in the ten experimental conditions (no secondary dimension was simulated). Mean and standard deviation are over 100 replications in each condition.

One convenient way to depict the size of bias in item discrimination in the designed conditions, for example, is by comparing it to the average size of item discrimination: Because an item discrimination value of 1 is considered an average item discrimination (Baker, 2001), the size of bias in condition 1 (bias $=0.007$ ) can be described as " $0.7 \%$ of the average item discrimination 
value", whereas the size of bias in condition 4 (bias $=0.384)$ can be described as " $38.4 \%$ of the average item discrimination". Because bias was positive, these values indicate how much the item discrimination parameter on the dominant dimension, on average (across 15 items), was overestimated in the unidimensional estimation in the given conditions.

Regarding RMSE (overall error) in estimation of item discrimination parameter (Table 3), the same pattern of results was found as in the case of bias: Overall error in the dominant dimension item discrimination parameter estimates was increasing with increase in strength of the secondary dimension. The magnitude of the relation between the strength of the secondary dimension and RMSE was 0.81 whereas the magnitude of the relation between the strength of the dominant dimension and RMSE was -0.02. The largest overall error was recorded in condition 4.

Table 3: Bias and RMSE in the Item Discrimination and Item Location Parameter Estimates in the Ten Designed Conditions

\begin{tabular}{|c|c|c|c|c|}
\hline \multirow{2}{*}{ Designed Conditions } & \multicolumn{2}{|c|}{ Item Discrimination } & \multicolumn{2}{|c|}{ Item Location } \\
\hline & $\begin{array}{c}\text { Bias } \\
M(S D)\end{array}$ & $\begin{array}{l}\text { RMSE } \\
M(S D)\end{array}$ & $\begin{array}{c}\text { Bias } \\
M(S D)\end{array}$ & $\begin{array}{l}\text { RMSE } \\
M(S D)\end{array}$ \\
\hline 1. Very strong - Negligible & $0.007(0.02)$ & $0.078(0.02)$ & $-0.058(0.01)$ & $0.134(0.01)$ \\
\hline 2. Very strong - Weak & $0.053(0.02)$ & $0.109(0.02)$ & $-0.102(0.01)$ & $0.274(0.01)$ \\
\hline 3. Very strong - Moderate & $0.187(0.02)$ & $0.278(0.02)$ & $-0.220(0.01)$ & $0.489(0.01)$ \\
\hline 4. Very strong - Strong & $0.384(0.02)$ & $0.493(0.01)$ & $-0.245(0.01)$ & $0.613(0.01)$ \\
\hline 5. Strong - Negligible & $0.009(0.01)$ & $0.059(0.01)$ & $-0.085(0.01)$ & $0.183(0.01)$ \\
\hline 6. Strong - Weak & $0.072(0.02)$ & $0.114(0.02)$ & $-0.139(0.01)$ & $0.349(0.01)$ \\
\hline 7. Strong - Moderate & $0.250(0.02)$ & $0.341(0.02)$ & $-0.275(0.01)$ & $0.593(0.01)$ \\
\hline 8. Moderate - Negligible & $0.015(0.01)$ & $0.055(0.01)$ & $-0.095(0.01)$ & $0.232(0.01)$ \\
\hline 9. Moderate - Weak & $0.100(0.01)$ & $0.144(0.01)$ & $-0.145(0.01)$ & $0.477(0.02)$ \\
\hline 10. Weak - Negligible & $0.027(0.01)$ & $0.065(0.01)$ & $-0.216(0.03)$ & $0.449(0.03)$ \\
\hline
\end{tabular}

Note: Mean and standard deviations for bias and RMSE are across 100 replications in each condition.

The results pertaining to bias in the item location estimation (Table 3) follow a similar pattern as the results obtained regarding bias in item discrimination estimation, with some differences noted. The relation between bias in item location and the strength of the secondary dimension was very strong - a correlation coefficient of 0.91 was recorded, a similar magnitude as in the case of item discrimination. A greater magnitude of the correlation between bias and the strength of the dominant dimension, after controlling for the strength of the secondary dimension, was recorded compared to the correlation obtained in case of item discrimination (correlation coefficient of 0.54$)$. 
About the size of bias, item location was underestimated for less than 0.2 SD in most of the conditions. Bias was larger in conditions 4, 7, and 3 (with a strong and a moderate secondary dimension), as well as in condition 10 (weak-negligible condition), but without exceeding 0.3 SD points in any of the conditions. In all the study conditions, bias in the item location estimates was negative, indicating underestimation of the item location parameter ${ }^{7}$. In relation to RMSE in unidimensional estimation of the item location parameter, the magnitude of the relation between the strength of the secondary dimension and RMSE was 0.97, whereas the magnitude of the relation between the strength of the dominant dimension and RMSE was -0.49 . Overall, in comparison to item discrimination estimates, greater contribution of the dominant dimension was noted to the item location estimation accuracy. The largest recorded overall error in item location estimates was about 0.6 SD.

\section{Person Location Parameter Estimation}

In relation to the person location estimation, in strictly unidimensional conditions bias in the unidimensional estimates of the dominant trait person location was very close to zero, as in case of bias in the item parameters estimates (Table 4). Different from the item parameters estimation, bias was not found in the unidimensional person location estimates in the ten designed conditions of unidimensionality violation - the average bias was very close to zero in each of the conditions, suggesting that there was no systematic distortion in the unidimensional estimates of the dominant latent trait person location in the ten conditions (Table 5). However, overall error, as indicated by RMSE, varied substantially across the conditions - it was increasing with increase in strength of the secondary dimension and with decrease in strength of the dominant dimension. The magnitude of the relation between the strength of the secondary dimension and RMSE was 0.80, whereas the magnitude of the relation between the strength of the dominant dimension and RMSE was -0.82 .

Further examination, at different levels of person location, shed more light on bias in person location parameter. Bias was summarized at three levels of latent trait: a) low range of latent trait, with person location parameter $\leq-1, \mathrm{~b}$ ) moderate range of latent trait, with person location parameter from -1 to 1 , and c) higher range of latent trait, with person location parameter $\geq 1$. The results revealed that the findings about no bias applied only to the moderate range of latent trait, in all of the conditions. At the lower range of latent trait, however, as well as at the higher range of latent trait, there was systematic error present in the estimates - in strictly unidimensional conditions (Table 4) as well as in each of the ten designed conditions (Table 5).

The results consistently indicated overestimation of the person location parameter at the lower range of the trait, and underestimation at the higher range of the trait. There was increase in bias

\footnotetext{
${ }^{7}$ It should be noted that, in accordance with the research design, only one set of parameters for the two dimensions was generated for all conditions (randomly generated from a standard normal distribution, in two successive draws). Bias in item location parameter estimates is related to the specific set of parameters, and its direction may vary with the different distributions of the item location parameters on the two dimensions (Finch, 2011).
} 
in the person location estimates, at both lower and higher ranges of latent trait, with increase in strength of the secondary dimension and with decrease in the strength of the dominant dimension. The magnitude of the correlation between the strength of the dominant dimension and bias at the low level of latent trait was 0.97 and in terms of bias at the high level of latent trait, the magnitude of the correlation was 0.93 (negative relations - bias increased with decrease in the strength of the dominant dimension), after controlling for the strength of the secondary dimension. The magnitude of the correlation between the strength of the secondary dimension and bias at the low level of latent trait was 0.59 and at the high level of latent trait, the magnitude of the correlation was 0.69 .

Table 4: Bias and RMSE in the Person Location Parameter Estimates in Strictly Unidimensional Conditions

\begin{tabular}{lccccc}
\hline \multicolumn{1}{c}{$\begin{array}{c}\text { Latent Trait } \\
\text { Strength }\end{array}$} & Bias & RMSE & \multicolumn{3}{c}{ Bias at Different Level of $\boldsymbol{\theta}$} \\
\cline { 4 - 6 } & & & Low $\boldsymbol{\theta}$ & Medium $\boldsymbol{\theta}$ & High $\boldsymbol{\theta}$ \\
\hline Very strong & 0.001 & 0.377 & 0.207 & 0.003 & -0.218 \\
\hline Strong & 0.001 & 0.430 & 0.300 & -0.005 & -0.276 \\
\hline Moderate & 0.001 & 0.524 & 0.433 & -0.006 & -0.404 \\
\hline Weak & 0.001 & 0.725 & 0.798 & 0.000 & -0.797 \\
\hline
\end{tabular}

Note: In strictly unidimensional conditions, no secondary dimension was simulated. $\theta=$ person location parameter. Reported values are $M$ across 100 replications in each condition ( $S D$ for bias and RMSE was $\leq 0.01$ in all conditions). The last three columns are mean bias across different levels of person location. Low $\theta: \theta \leq-1$; Medium $\theta:-1<\theta<1$; High $\theta: \theta \geq 1$.

In terms of the size of bias, in most of the conditions bias was less than $0.5 \mathrm{SD}$; that is, the person location was overestimated in the case of the low trait and underestimated for the high level of trait for less than $0.5 \mathrm{SD}$ points ${ }^{8}$. In one condition (condition 10), bias exceeded 0.8 SD points. In this condition, the dominant factor was "weak" - such a condition would be unlikely in measures in practical use, as the solution with the dominant factor of such strength would be flagged as problematic in the traditional, commonly performed dimensionality assessment.

In relation to interpreting the magnitude of bias, it is important to state that answering the question whether bias is "small" or "large" is tied to the use of the measure and the practical consequences that may result from the use of the distorted estimates. For different practical uses, a different amount of distortion in IRT model estimates of person location may be relevant (Zhao

\footnotetext{
${ }^{8}$ It should be noted, in relation to the size of error in person location parameter estimates, a certain amount of bias was recorded even in the strictly unidimensional condition, indicating that the contributing effects of the length of the measure (15 items were used in this study) and estimation method should be looked into further. Because shorter measures are common in general psychological measurement and research, the study findings pointed to a need for more details about IRT application to such measures.
} 
\& Hambleton, 2017). In the context of real-life measures, the magnitude of error in the estimates should be interpreted in the specific practical contexts. Providing more detailed information about error at different levels of violation of unidimensionality, rather than interpreting its magnitude and relevance, was a guiding idea for this research.

Table 5: Bias and RMSE in the Person Location Parameter Estimates in the Ten Designed Conditions

\begin{tabular}{lccccc}
\hline \multirow{1}{*}{ Designed Conditions } & \multirow{2}{*}{ Bias } & \multirow{2}{*}{ RMSE } & \multicolumn{3}{c}{ Bias at Different Level of $\theta$} \\
\cline { 4 - 6 } & & & Low $\boldsymbol{\theta}$ & Medium $\boldsymbol{\theta}$ & High $\boldsymbol{\theta}$ \\
\hline 1. Very strong - Negligible & $<0.001$ & 0.386 & 0.211 & 0.004 & -0.230 \\
\hline 2. Very strong - Weak & $<0.001$ & 0.439 & 0.242 & 0.005 & -0.262 \\
\hline 3. Very strong - Moderate & $<0.001$ & 0.560 & 0.323 & 0.008 & -0.358 \\
\hline 4. Very strong - Strong & $<0.001$ & 0.679 & 0.421 & 0.011 & -0.466 \\
\hline & & & & & \\
\hline 5. Strong - Negligible & $<0.001$ & 0.445 & 0.301 & -0.002 & -0.292 \\
\hline 6. Strong - Weak & $<0.001$ & 0.514 & 0.345 & -0.001 & -0.340 \\
\hline 7. Strong - Moderate & $<0.001$ & 0.658 & 0.454 & 0.002 & -0.461 \\
\hline & & & & & \\
\hline 8. Moderate - Negligible & $<0.001$ & 0.541 & 0.435 & -0.001 & -0.427 \\
\hline 9. Moderate - Weak & $<0.001$ & 0.626 & 0.494 & 0.000 & -0.493 \\
\hline & & & & & \\
\hline 10. Weak - Negligible & $<0.001$ & 0.747 & 0.803 & 0.004 & -0.821 \\
\hline
\end{tabular}

Note: $\theta=$ person location parameter. Reported values are $M$ across 100 replications in each condition ( $S D$ for bias and RMSE was $\leq 0.01$ in all conditions). The last three columns are mean bias across different levels of person location. Low $\theta$ : $\theta \leq-1$; Medium $\theta$ : $-1<\theta<1$; High $\theta: \theta \geq 1$.

\section{Discussion}

In the current study, different levels of deviations from strict unidimensionality and the accuracy in unidimensional latent variable models estimates were further investigated, in particular types of violations. As opposed to unidimensionality violations when the measure captures more than one interpretable construct, of interest were conditions when all of the items primarily capture a single construct (as intended in a unidimensional measure), but they also, to a smaller degree, tap into some additional influences. Among the additional influences that can be affecting item responding are various respondents' characteristics, such as background characteristics (e.g., gender and ethnicity), as indicated in a number of measurement invariance and differential item functioning studies. Personality characteristics such as anxiety, introspectiveness, perfectionism, self-confidence, and their combinations can influence measurements when another specific trait or ability is intended to be measured. Respondents' motivation, concentration/focusing ability, and sensitivities to various situational factors can be relevant for the measure responding. Individual and group differences in the characteristics such as those listed can be, depending on the type and purpose of the measurement, captured by our measures and could be impacting the 
measurement outcomes. In addition to the characteristics of the respondents, characteristics of the items and various method effects can affect measurement outcomes, as studied in a large body of psychometric research.

The model and study design utilized in the current study (a two-dimensional model with complex-structure items, and a research design based on manipulating the strength of the latent dimensions in a particular way) were proposed as useful for studying different levels of deviation from strict unidimensionality in measures intended and designed as unidimensional. In the several simulated composites of a dominant latent dimension (reflected by all the items) and a smaller secondary nuisance dimension, the accuracy in the unidimensional estimates was explored. Specifically, overall error and bias in the unidimensional item and person estimates regarding the dominant latent trait (i.e., unidimensional measurement target) were examined.

In the context of the specific unidimensional model assessed (2PL IRT model), various degrees of systematic error (bias) were demonstrated in the unidimensional item and person parameters estimation (e.g., overestimated item discrimination/factor loadings). The bias increased with increase in the strength of the secondary dimension while controlling for the strength of the dominant dimension. In terms of item discrimination (factor loadings) for example, a very strong relation was demonstrated between the size of bias in item discrimination and the strength of the secondary dimension (correlation of 0.90), and a weak relation with the strength of the dominant dimension ${ }^{9}$. The importance of the strength of the dominant dimension increased when item location estimation was in question, and particularly, person location estimation. The details about the strength of the examined relations, and about the size of the bias and overall error in the unidimensional item and person location parameter estimates, due to different levels of unidimensionality violations, were provided in the Results section.

In the conditions addressed in the current study, item discrimination index/factor loadings were systematically overestimated, consistent with the previous findings of the research concerned with the effects of various types of LI violations (Sireci et al., 1991; Wainer \& Thissen, 1996). Overestimation of the item discrimination parameter indicates overestimation of the measurement precision (e.g., inflated reliability and item/test information function in FA and IRT), leading to inflation in researchers' confidence in the conclusions based on the use of the measure. Varying degrees of factor loadings overestimation and the related measurement error underestimation, if unknown/unreported, are important, and can impact the results/conclusions of substantive research and applied measurement (e.g., their replicability) when the unidimensional model estimates are used. Varying degrees of bias in item parameters estimates can influence other analyses that use these estimates (e.g., structural equation modeling, SEM, analyses) and various applications specific for IRT (e.g., selection of items in computer adaptive testing). In relation to the former, some of the effects of the distortions in the measurement model estimates on the accuracy of the structural coefficients in the SEM were pointed to in Reise et al. (2013), whereas in relation to the latter, a need for more detailed investigation was recommended in Folk

\footnotetext{
${ }^{9}$ Even with a very strong dominant dimension, there could be substantial bias in unidimensional item discrimination/factor loadings estimates resulting from the additional, unintendedly captured influences.
} 
and Green (1989) and Zhao and Hambleton (2017). Additionally, the process of dimensionality assessment and the decisions about whether the measure is unidimensional (i.e., unidimensional enough) can be affected when latent variable models estimates are used for such a purpose.

In terms of the size of bias in item parameters, in a range of conditions the bias could be seen as "small" (as in the conditions with a negligible secondary dimension in this study), and possibly without a substantial impact on some of the practical uses of the estimates. However, this does not have to necessarily be the case. Bias was systematically increasing with increase in the strength of the secondary dimension, and it can be substantial, despite a very strong dominant dimension. This is the case when "something else" - another source of item dependencies, or a group of related sources, is emerging in item responses (e.g., depicted in conditions 3 and 4 in this study). "Rising" multidimensionality in a unidimensional measure and the resulting bias in the unidimensional estimation, when not identified, are problematic.

When IRT estimation of person location is of interest, bias in the estimates of target person location parameter was recorded at more extreme ends of the latent trait; specifically, overestimation at the lower end and underestimation at the higher end of the latent dimension. The consequences of varying degrees of bias at the more extreme ends of the latent trait can be important, for instance, when the cut-points are used at the more extreme ends of the distribution (such as for the purpose of screening or diagnostic decisions). For bias and overall error in the unidimensional person location parameter, the strength of both the dimensions is of importance they were systematically increasing with both increase in the strength of the secondary dimension and with decrease in the strength of the dominant dimension.

Overall, as it has been demonstrated in psychometric research (e.g., in Reckase, Ackerman, \& Carlson, 1988), in some circumstances when the items in measures capture composites of latent dimensions rather than a single dimension, the measures still can be seen as unidimensional, and a one-factor model could fit the data well (based on the FA model-fit and local dependence indices). When hybrids of the latent traits are captured, mathematical unidimensionality may be met $^{10}$. However, in such conditions, there is a certain amount of systematic distortions in the unidimensional model estimates regarding the dominant latent trait (i.e., unidimensional measurement target). Different degrees of bias in the unidimensional item and person parameter estimates, due to different levels of deviation from strict unidimensionality, were demonstrated in the current study. Varying degrees of bias (when one-factor model may be demonstrating a good fit) can be problematic - when the bias is unreported, problems can arise in relation to the reliability of measurement (i.e., unknown/distorted measurement error), validity of measurement

\footnotetext{
${ }^{10}$ In the current study, the conducted supplementary analyses confirmed that "unidimensionality" was met, and that one-factor model demonstrated a good fit to the data in the designed composites. Specifically, CFA with ordinal item responses was conducted, by utilizing the "lavaan' R package (Rosseel, 2012) and the recommended estimation (Muthen, du Toit, \& Spisic, 1997). The analyses confirmed that in each of the ten conditions the unidimensional model demonstrated a good fit to the data: In 100 replications in each of the ten designed conditions, the highest mean SRMS was 0.03; the mean RMSEA was $<0.01$; the mean GFI and CFI were $\geq 0.99$.
} 
(e.g., measures could be declared as unidimensional sometimes and other times not, resulting in the questions about what it is that our measures really measure), as well as in relation to the fairness of measurements (e.g., when bias is affecting certain groups/individuals). When convenient traditional unidimensional latent variable models are used, providing information about the size of systematic distortions in the estimates due to different levels of deviation from strict unidimensionality is needed.

The use of the model/design employed in the current study contributed further information to the findings of the previous research concerned with more detailed mapping and communicating error in the unidimensional estimates due to unidimensionality violations (e.g., Bonifay et al., 2015; Reise et al., 2013). The multidimensional IRT model employed in the study is among the models that can be seen, within the latent variable framework, as more realistic representation of the item responding processes in the measures intended as unidimensional. When, for convenience reasons, traditional unidimensional latent variable models are employed, this model could be used for providing more information about systematic distortions in the unidimensional estimation. A multidimensional model with two dimensions was proposed as sufficient for such a purpose, compared to more complex models (various multidimensional models with multiple secondary dimensions). Such a within-item multidimensional model with two dimensions could be estimated from the data in different ways (e.g., Ip, 2010; Ip \& Chen, 2012; Jennrich \& Bentler, 2011; Kahraman, 2013; Reckase, 2009; Reise et al., 2007; Rijmen, 2010, etc.), with the best way of estimation under investigation, and the guidelines to be provided. By comparing the model item and person estimates on the dominant dimension to the unidimensional model estimates, as performed in this study, information about the size of systematic distortions in the unidimensional estimation can be obtained.

In conclusion, assumption of unidimensionality is an idealization (McDonald, 1999; Nandakumar, 1991; Reckase, 2009; Stout, 1987; Traub, 1983) that cannot be met in measurement in the realms of human psychology, behavior, health, education, employment, etc. Various influences, in addition to the targeted trait, can be reflected by the items responses in particular measures and particular contexts. Unidimensional latent variable models estimates are affected by such influences - aside from random measurement error, systematic distortions in the estimates can occur. When systematic distortions in widely used measurement models are uncaptured/uncommunicated, various negative consequences can ensue for applied research and for practical measurements (the reliability, validity, and fairness of measurements, as well as the process of knowledge generation and knowledge synthesis can be impacted). In the current study, further information about bias possible in the unidimensional latent variable model item and person parameter estimates has been provided. As opposed to one-size-fits-all recommendations about when bias is "small enough", the size of bias was provided, needed for making informed decisions about appropriateness of the use of the unidimensional estimates in the particular contexts and practical applications ${ }^{11}$. The exploratory approach utilized in the study is recommended as a beneficial addition to predominant confirmatory approaches in

\footnotetext{
${ }^{11}$ Interpreting the size and importance of measurement error should be performed in relation to the types of decisions and the seriousness of possible consequences resulting from the use of the relevant estimates.
} 
providing further information about violations of strong measurement assumptions and their consequences, and in ensuring robust and valid measurements and research outcomes in social and behavioural sciences.

\section{Limitations and Future Directions}

The current study is a simulation study - the data are simulated to depict particular conditions of unidimensionality violations, in order to look into the possible effects of the violations and into the strength of the relevant relations. Only several points of the strength of the underlying dimension, out of the possible values, were selected in creating different conditions of deviations from strict unidimensionality, that is, "levels" rather than "degrees" of unidimensionality were investigated. The selected points were based on the criteria utilized in the IRT literature (Baker, 2001), and the ten conditions of violations were created as an appropriate start in the proposed research direction. As the simulation procedure was designed to depict certain type of violations, indicators that may reflect substantive multidimensionality in a measure were avoided. Inclusion of such indicators and a greater variability of the discrimination parameters should be further studied, as well as other sets of conditions that may be found in the weaker type of unidimensionality violations (only a particular set of conditions was addressed in the current study). The current investigation was conducted with normally distributed latent traits, as the first step in the given research direction.

The accuracy of the particular unidimensional model was examined in this study (i.e., a twoparameter IRT model). Due to the mathematical relation between two-parameter IRT models with ordinal FA models, the findings regarding error in item parameters estimates are generalizable to such contexts (e.g., to the ordinal CFA factor loadings estimates). The findings should not be generalized to other IRT models (e.g., one-parameter IRT models). Different models for binary and polytomous item responses and different estimation methods need further examination, in combination with the effects of measure length and sample sizes. Of interest in the current study was the type of violation of strict unidimensionality in measures intended as unidimensional, when some difficult to interpret secondary dimension(s) underlie the item responses in addition to the dominant dimension. In such a case, it may be worthwhile to try to keep the convenience of the unidimensional model, while providing adequate information about the distortions in the unidimensional parameter estimates. The problems addressed in the study are also relevant for multidimensional measures (intended to capture more than one specific construct) - more evidence about the effects of unintendedly captured influences on the multidimensional latent variable models estimates is needed.

Supplementary materials for this manuscript can be found at https://osf.io/htcxe/quickfiles (Supplemental Materials file)

The current research was supported by the Social Sciences and Humanities Research Council funding. I thank B. D. Zumbo, B. F. French, A. M. Hubley, A. Wu, and C. G. Richardson for their review of the performed analyses and for their feedback on the first draft of this manuscript. 


\section{References}

Ackerman, T. A. (1989). Unidimensional IRT calibration of compensatory and noncompensatory multidimensional items. Applied Psychological Measurement, 13, 113-127. doi:10.1177/014662168901300201

Ansley, T. N., \& Forsyth, R. A. (1985). An examination of the characteristics of unidimensional IRT parameter estimates derived from two-dimensional data. Applied Psychological Measurement, 9, 37-48. doi: 10.1177/014662168500900104

Baker, F. (2001). Basics of item response theory. Retrieved from http://echo.edres.org:8080/irt/baker

Bock, R. D. (1972). Estimating item parameters and latent ability when responses are scored in two or more nominal categories. Psychometrika, 37, 29-51. doi: 10.1007/BF02291411

Bonifay, W. E., Reise, S. P., Scheines, R., \& Meijer, R. R. (2015). When are multidimensional data unidimensional enough for structural equation modeling? An evaluation of the DETECT multidimensionality index. Structural Equation Modeling: A Multidisciplinary Journal, 22, 504-516. doi:10.1080/10705511.2014.938596

Birnbaum, A. (1968). Some latent trait models. In F. M. Lord \& M. R. Novick (Eds.), Statistical theories of mental test scores (pp. 397-424). Reading, MA: Addison-Wesley.

Chalmers, R. P. (2012). mirt: A multidimensional item response theory package for the R environment. Journal of Statistical Software, 48, 1-29. doi:10.18637/jss.v048.i06

Chen, W. H., \& Thissen, D. (1997). Local dependence indexes for item pairs using item response theory. Journal of Educational and Behavioral Statistics, 22, 265-289. doi: $10.2307 / 1165285$

Crişan, D. R., Tendeiro, J. N., \& Meijer, R. R. (2017). Investigating the practical consequences of model misfit in unidimensional IRT models. Applied Psychological Measurement, 41, 439-455. doi: 10.1177/0146621617695522

de Ayala, R. J. (2009). The theory and practice of item response theory. New York, NY: Guilford Press.

DeMars, C. E. (2012). Confirming testlet effects. Applied Psychological Measurement, 36, 104 121. doi.org/10.1177/0146621612437403

Drasgow, F., \& Parsons, C. K. (1983). Application of unidimensional item response theory models to multidimensional data. Applied Psychological Measurement, 7, 189-199. doi/10.1177/014662168300700207

Embretson, S. E., \& Reise, S. P. (2000). Item response theory for psychologists. Mahwah, NJ: Lawrence Erlbaum.

Feinberg, R. A., \& Rubright, J. D. (2016). Conducting simulation studies in psychometrics. Educational Measurement: Issues and Practice, 35, 36-49. doi.org/10.1111/emip.12111

Finch, H. (2011). Multidimensional item response theory parameter estimation with nonsimple structure items. Applied Psychological Measurement, 35(1), 67-82.

Folk, V. G., \& Green, B. F. (1989). Adaptive estimation when the unidimensionality assumption of IRT is violated. Applied Psychological Measurement, 13, 373-389. doi:10.1177/014662168901300404

Hambleton, R. K., \& Swaminathan, H. (1985). Item response theory: Principles and applications. Boston: Kluwer. 
Harwell, M., Stone, C. A., Hsu, T. C., \& Kirisci, L. (1996). Monte Carlo studies in item response theory. Applied Psychological Measurement, 20, 101-125. doi:10.1177/014662169602000201

Hattie, J. (1985). Methodology review: assessing unidimensionality of tests and items. Applied Psychological Measurement, 9, 139-164. doi:10.1177/014662168500900204

Huber, P. J. (1981). Robust statistics. New York: Wiley.

Ip, E. H. (2010). Empirically indistinguishable multidimensional IRT and locally dependent unidimensional item response models. British Journal of Mathematical and Statistical Psychology, 63, 395-416. doi: 10.1348/000711009X466835

Ip, E. H. S., \& Chen, S. H. (2012). Projective item response model for test-independent measurement. Applied Psychological Measurement, 36, 581-601. doi: $10.1177 / 0146621612452778$

Jennrich, R. I. , \& Bentler, P. (2011). Exploratory bi-factor analysis. Psychometrika , 76 (4), 537-549. https://doi.org/10.1007/s11336-011-9218-4

Jöreskog, K. G. (1969). A general approach to confirmatory maximum likelihood factor analysis. Psychometrika, 34, 183-202.

Kahraman, N. (2013). Unidimensional interpretations for multidimensional test items. Journal of Educational Measurement, 50, 227-246. doi:10.1111/jedm.12012

Kamata, A., \& Bauer, D. J. (2008). A note on the relation between factor analytic and item response theory models. Structural Equation Modeling, 15, 136-153. doi: $10.1080 / 10705510701758406$

Kirisci, L., Hsu, T. C., \& Yu, L. (2001). Robustness of item parameter estimation programs to assumptions of unidimensionality and normality. Applied Psychological Measurement, 25, 146-162. doi:10.1177/01466210122031975

Lazarsfeld, P. F. (1950). The logical and mathematical foundation of latent structure analysis. In S. A. Stouffer, L. Guttman, E. A. Suchman, P. F. Lazarsfeld, S. A. Star, \& J. A. Clausen (Eds.), Measurement and prediction (pp. 362-412). New York, NY: Wiley.

Lord, F. M. (1952). A theory of test scores. Psychometric Monograph No. 7. Richmond, VA: Psychometric Corporation. Retrieved from: http://www.psychometrika.org/journal/online/MN07.pdf

Lord, F. M., \& Novick, M. R. (1968). Statistical theories of mental test errors. Reading, MA: Addison-Wesley.

McDonald, R. P. (1981). The dimensionality of tests and items. British Journal of Mathematical and Statistical Psychology, 34, 100-117.

McDonald, R. P. (1999). Test theory: A unified treatment. Mahwah, NJ: Erlbaum.

McDonald, R. P. (2000). A basis for multidimensional item response theory. Applied Psychological Measurement, 24, 99-114. doi:10.1177/01466210022031552

Millsap, R.E. (2011). Statistical approaches to measurement invariance. Routledge, New York, NY.

Morin, A. J., Arens, A. K., Tran, A., \& Caci, H. (2016). Exploring sources of construct-relevant multidimensionality in psychiatric measurement: A tutorial and illustration using the Composite Scale of Morningness. International Journal of Methods in Psychiatric Research, 25(4), 277-288.

Nandakumar, R. (1991). Traditional dimensionality versus essential dimensionality. Journal of Educational Measurement, 28, 99-117. doi: 10.1111/j.1745-3984.1991.tb00347.x 
Nandakumar, R., \& Stout, W. (1993). Refinements of Stout's procedure for assessing latent trait unidimensionality. Journal of Educational Statistics, 18, 41-68. doi: $10.3102 / 10769986018001041$

R Development Core Team (2019). R: A language and environment for statistical computing. Vienna, Austria: R Foundation for Statistical Computing. Available from https://www.Rproject.org/

Rajlic, G., Kwon, J. Y., Roded, K., \& Hubley, A. M. (2019). Development of the Global SelfEsteem Measure: A pilot study. Journal of Psychoeducational Assessment, 37(7), 863873. doi:10.1177/0734282918801816

Reckase, M. D. (1979). Unifactor latent trait models applied to multifactor tests: Results and implications. Journal of Educational Statistics, 4, 207-230. doi: 10.3102/10769986004003207

Reckase, M. D. (2009). Multidimensional item response theory. New York, NY: Springer.

Reckase, M. D., Ackerman, T. A., \& Carlson, J. E. (1988). Building a unidimensional test using multidimensional items. Journal of Educational Measurement, 25(3), 193-203.

Reise, S. P., Morizot, J., \& Hays, R. D. (2007). The role of the bifactor model in resolving dimensionality issues in health outcomes measures. Quality of Life Research, 16, 19 - 31.

Reise, S. P., Scheines, R., Widaman, K. F., \& Haviland, M. G. (2013). Multidimensionality and structural coefficient bias in structural equation modeling: A bifactor perspective. Educational and Psychological Measurement, 73, 5-26. doi: 10.1177/0013164412449831

Reise, S. P., \& Waller, N. G. (2009). Item response theory and clinical measurement. Annual Review of Clinical Psychology, 5, 27-48.

Rijmen, F. (2010). Formal relations and an empirical comparison among the bi-factor, the testlet, and a second-order multidimensional IRT model. Journal of Educational Measurement, 47, 361-372. doi:10.1111/j.1745-3984.2010.00118.x

Rizopoulos, D. (2006). 1tm: An R package for latent variable modeling and item response theory analyses. Journal of Statistical Software, 17, 1-25. doi: 10.18637/jss.v017.i05

Samejima, F. (1969). Estimation of latent ability using a response pattern of graded scores (Psychometric Monograph No. 17). Richmond, VA: Psychometric Society. Retrieved from http://www.psychometrika.org/journal/online/MN17.pdf

Sireci, S. G., Thissen, D., \& Wainer, H. (1991). On the reliability of testlet-based tests. Journal of Educational Measurement, 28, 237-247.

Steinberg, L., \& Thissen, D. (1996). Uses of item response theory and the testlet concept in the measurement of psychopathology. Psychological Methods, 1, 81-97. doi: 10.1037/1082989X.1.1.81

Stout, W. (1987). A nonparametric approach for assessing latent trait unidimensionality. Psychometrika, 52, 589-617. doi: 10.1007/BF02294821

Takane, Y., \& De Leeuw, J. (1987). On the relationship between item response theory and factor analysis of discretized variables. Psychometrika, 52, 393-408. doi: 10.1007/BF02294363

Traub, R. (1983). A prior consideration in choosing an item response model. In R. K. Hambleton (Ed.), Applications of Item Response Theory. Vancouver, BC: Educational Research Institute of British Columbia.

Wainer, H., \& Thissen, D. (1996). How is reliability related to the quality of test scores? What is the effect of local dependence on reliability? Educational Measurement: Issues and Practice, 15, 22-29. doi: 10.1111/j.1745-3992.1996.tb00803.x 
Wirth, R. J., \& Edwards, M. C. (2007). Item factor analysis: current approaches and future directions. Psychological Methods, 12, 58-79. doi: 10.1037/1082-989X.12.1.58

Yen, W. M. (1993). Scaling performance assessments: Strategies for managing local item dependence. Journal of Educational Measurement, 30, 187-213. doi: 10.1111/j.17453984.1993.tb00423.x

Zhao, Y., \& Hambleton, R. K. (2017). Practical consequences of item response theory model misfit in the context of test equating with mixed-format test data. Frontiers in Psychology, 8, 484. doi: 10.3389/fpsyg.2017.00484

Zhang, B. (2008). Application of unidimensional item response models to tests with items sensitive to secondary dimensions. The Journal of Experimental Education, 77, 147-166. 